\title{
Enhanced Electromechanical Response in Sm and Nd Co-doped Ceria
}

\author{
Kabir, Ahsanul; Bowen, Jacob R.; Varenik, Maxim; Lubomirsky, Igor; Esposito, Vincenzo
}

Published in:

Acta Materialia

Link to article, DOI:

10.1016/j.mtla.2020.100728

Publication date:

2020

Document Version

Peer reviewed version

Link back to DTU Orbit

Citation (APA):

Kabir, A., Bowen, J. R., Varenik, M., Lubomirsky, I., \& Esposito, V. (2020). Enhanced Electromechanical Response in Sm and Nd Co-doped Ceria. Acta Materialia, 12, [100728].

https://doi.org/10.1016/j.mtla.2020.100728

\section{General rights}

Copyright and moral rights for the publications made accessible in the public portal are retained by the authors and/or other copyright owners and it is a condition of accessing publications that users recognise and abide by the legal requirements associated with these rights.

- Users may download and print one copy of any publication from the public portal for the purpose of private study or research.

- You may not further distribute the material or use it for any profit-making activity or commercial gain

- You may freely distribute the URL identifying the publication in the public portal

If you believe that this document breaches copyright please contact us providing details, and we will remove access to the work immediately and investigate your claim. 


\section{Journal Pre-proof}

\section{Enhanced Electromechanical Response in Sm and Nd Co-doped}

Ceria

Ahsanul Kabir, Jacob R. Bowen, Maxim Varenik, Igor Lubomirsky, Vincenzo Esposito

PII: S2589-1529(20)30145-9

DOI: https://doi.org/10.1016/j.mtla.2020.100728

Reference: MTLA 100728

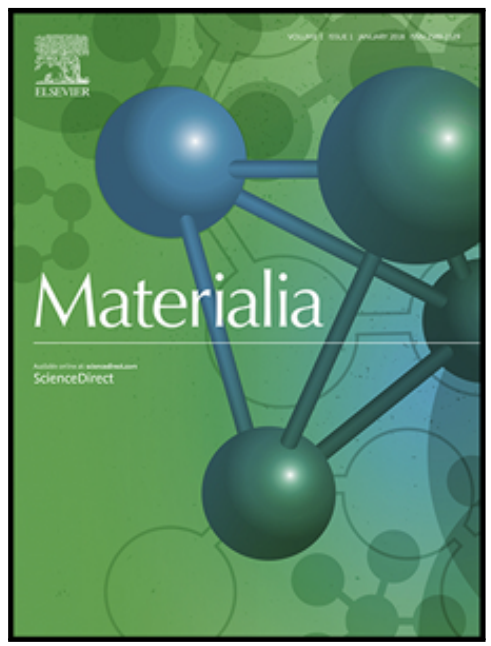

To appear in:

Materialia

Received date: 3 February 2020

Accepted date: 15 May 2020

Please cite this article as: Ahsanul Kabir, Jacob R. Bowen, Maxim Varenik, Igor Lubomirsky, Vincenzo Esposito, Enhanced Electromechanical Response in Sm and Nd Co-doped Ceria, Materialia (2020), doi: https://doi.org/10.1016/j.mtla.2020.100728

This is a PDF file of an article that has undergone enhancements after acceptance, such as the addition of a cover page and metadata, and formatting for readability, but it is not yet the definitive version of record. This version will undergo additional copyediting, typesetting and review before it is published in its final form, but we are providing this version to give early visibility of the article. Please note that, during the production process, errors may be discovered which could affect the content, and all legal disclaimers that apply to the journal pertain.

(C) 2020 Published by Elsevier B.V. on behalf of Acta Materialia Inc. 


\section{Enhanced Electromechanical Response in Sm and Nd Co- doped Ceria}

Ahsanul Kabir ${ }^{1}$, Jacob R. Bowen ${ }^{1}$, Maxim Varenik ${ }^{2}$, Igor Lubomirsky ${ }^{2}$, Vincenzo Esposito ${ }^{1^{*}}$

${ }^{1}$ Department of Energy Conversion and Storage, Technical University of Denmark (DTU), Kgs. Lyngby 2800, Denmark

${ }^{2}$ Department of Materials and Interfaces, Weizmann Institute of Science (WIS), Rehovot 76100, Israel

*Corresponding Authors: E-mail: ahsk@dtu.dk, vies@dtu.dk

Keywords: co-doped ceria, oxygen vacancies, ionic conductivity, blocking barriers, electrostriction 


\section{Abstract}

Highly oxygen defective cerium oxide, e.g., Gd-doped ceria, is a sustainable non-classical electrostrictor with electromechanical properties that are superior to lead-based piezoelectric metal oxides. Here, we report electrostriction in co-doped ceria ( $\mathrm{Sm}, \mathrm{Nd})$ with a nominally low short-range vacancy-dopant association energy. Such a strategy results in a higher electrostrictive strain coefficient $\left(\mathrm{M}_{33}\right)$, up to $10^{-17}(\mathrm{~m} / \mathrm{V})^{2}$ at lower-frequencies, and unexpected electromechanical strain saturation and relaxation effects. These outcomes support the hypothesis that electrostriction is strongly influenced by the local environment of oxygen vacancy and by the ionic migration blocking factors built-in the microstructure. 


\section{Introduction}

Electrostrictive materials are subjected to a wide variety of technological applications as actuators in mechanics, electronics, and biomedical devices [1][2][3]. Electrostriction is the electromechanical response that arises in electrostrictors as the second-order elastic deformation under an applied electric field [1][4]. It is present in all dielectrics regardless of crystal structure and is described by a fourth-ranked tensor that follows the relationship [5],

$$
x_{i j}=M_{i j m n} E_{m} E_{n}
$$

Where $M_{i j m n}$ is the electrostrictive field related strain coefficient and $E$ is the external electric field. The values of $M_{i j m n}$ typically range (for inorganic compounds) from $\sim 10^{-21}-10^{-16}(\mathrm{~m} / \mathrm{V})^{2}$ that depends on both the dielectric permittivity and elastic modulus of the materials [1]. However, the electrostrictive strain is not always quadratic, as the dielectric constant often increases non-linearly with the electric field, especially in high permittivity materials. Accordingly, Newnham et al. proposed describing electrostrictive properties by means of polarization electrostriction coefficient $\left(Q_{i j m n}\right)$, which illustrates a better representation of the quadratic form of electrostriction, expressed as [1],

$$
x_{i j}=Q_{i j m n} P_{m} P_{n}
$$

Here, $\mathrm{P}$ is the dielectric polarization. Furthermore, to compare electrostrictive materials with different crystallographic symmetry a universal hydrostatic electrostriction polarization coefficient $\left(Q_{h}\right)$ has been formalized, in which $Q_{h}$ is associated with the dielectric permittivity $(\varepsilon)$ and inverse Young's modulus (S) in an empirical relation, given as [6],

$$
\left|\mathrm{Q}_{\mathrm{h}}\right| \approx 2.37 \cdot\left(\mathrm{S} / \varepsilon \varepsilon_{0}\right)^{0.59}
$$

(3)

Recently, it has been shown that thin films of defective cerium oxide, e.g., $20 \mathrm{~mol} \% \mathrm{Gd}$ doped ceria $(\mathrm{GDC}),\left(\varepsilon^{\mathrm{GDC}} \approx 30\right.$ and $\left.\mathrm{Y}^{\mathrm{GDC}} \approx 200 \mathrm{GPa}\right)$ exhibit giant electrostrictive strain 
coefficient $\left(\mathrm{M}_{33}\right)$ around $6.5 \cdot 10^{-18}(\mathrm{~m} / \mathrm{V})^{2}$ at $0.1 \mathrm{~Hz}$ [7]. The estimated $\left|\mathrm{Q}_{\mathrm{h}}\right|$ reveals at least two orders of magnitude larger than the Newnham scaling law in Eqn 3. Similar results have been reported for highly defective GDC and bismuth oxide-based bulk ceramics [6][8][9]. The atomistic mechanism functioning this non-classical type of electrostriction is due to the presence of electroactive $\mathrm{Ce}-\mathrm{O}$ bonds in the crystal lattice, as explained by Lubomirsky and co-workers [7][10][11]. Based on extended X-ray absorption fine structure (EXAFS) and Xray absorption near edge structure (XANES) experiments, it has been observed that oxygen vacancies $\left(\mathrm{V}_{\mathrm{O}}^{*}\right)$ in the lattice create a small population of distorted $\mathrm{Ce}_{\mathrm{Ce}_{\mathrm{e}}}-7 \mathrm{O}_{\mathrm{o}}-\mathrm{V}_{\mathrm{O}}^{\prime \prime}$ units, which consists of elongated $\mathrm{Ce}-\mathrm{V}_{\mathrm{O}}$ and contracted $\mathrm{Ce}-\mathrm{O}$ bonds in comparison with the Ce-O bond in the Ce- $80_{o}$ unit. This results in asymmetric local charge configuration and anisotropic local dipolar elastic strain around the lattice [10][12]. Hence, upon application of an electric field, these electroactive bonds change dynamically and become ordered, inducing a large macroscopic electromechanical displacement [10][12][13]. Other reports demonstrate that $\mathrm{M}_{33}$ in GDC bulk ceramics exhibits strain saturation and relaxation in response to the applied electric field and frequency, respectively [8][9][14]. To date, the electrostriction effect in defective ceria is investigated only with pure and Gd-doped ceria. Therefore, it is important to understand the role of other types of dopants, i.e., the physical correlation between dopant associated oxygen vacancy concentration and the electrostriction.

In the present work, we aim to optimize the electrostrictive properties by tuning the configuration of oxygen vacancy in the lattice by substituting $\mathrm{Gd}$ with a co-dopant combination of $\mathrm{Sm} / \mathrm{Nd}$ (1:1 ratio). Based on the computational prediction reported in previous work [15], an equimolar co-doped system with an effective atomic radius number around 61, i.e., ionic radius $1.093 \AA$, exerts lesser interaction between dopant and oxygen vacancy compared to a singly doped ceria compound. In such a case, oxygen vacancies show no site preferences, eventually resulting in an enhanced configurational entropy [15][16][17]. Although difficult to formalize, the configuration of oxygen vacancy can be macroscopically characterized by electrochemical impedance spectroscopy (EIS) [18][19]. 
This technique can resolve the interlinked effects between the dopants, oxygen vacancies concentration, and microstructure by separating the overall macroscopic electrochemical response into defined domains of electrical impedance, i.e., electrochemical capacitance and ionic migration blocking effects [20][21][22]. The ion blocking factor $\left(\alpha_{\text {g.b. }}\right)$ defined by EIS serves as a descriptor of the configuration of oxygen vacancy $\left(\mathrm{V}_{\mathrm{O}}^{*} / \mathrm{Ce}_{\mathrm{Ce}}-\mathrm{V}_{\mathrm{O}}^{\prime \prime}\right)$. The value of the $\alpha_{\text {g.b. }}$ at $300{ }^{\circ} \mathrm{C}$, is here correlated with the electromechanical response, in an attempt to clarify the interlinked effects between oxygen vacancy concentration and their configuration in doped ceria.

\section{Experimental Procedure}

Nanometer-scale Sm and Nd-co-doped ceria (CDC) powders with a composition of $\mathrm{Ce}_{1-\mathrm{x}} \mathrm{Sm}_{\mathrm{x} / 2} \mathrm{Nd}_{\mathrm{x} / 2} \mathrm{O}_{2-\mathrm{x} / 2}$ (where $\mathrm{x}=0.01,0.05,0.1$, and 0.15 referred to as CDC-1, CDC-5, CDC-10, and CDC-15, respectively) were synthesized by the co-precipitation method [20]. Metal nitrate salts (Sigma-Aldrich, USA) of the corresponding element were dissolved in a stoichiometric ratio in deionized water (0.1 M solution). Afterwards, N-methyl-diethanolamine was slowly added to the solution, keeping the $\mathrm{pH} \approx 10$. The precipitates were formed immediately and were kept overnight under slow stirring. Precipitates were then centrifuged and washed several times with ethanol, followed by calcination at $500{ }^{\circ} \mathrm{C}$ for 2 hours. Hereafter, the dried hard agglomerated powders were ball-milled in ethanol at $50 \mathrm{rpm}$ for 10 hours and subsequently dried at $120^{\circ} \mathrm{C}$. Finally, the powders were softly ground in an agate mortar pestle and sieved through a $150 \mu \mathrm{m}$ mesh. The powders were then cold-pressed as pellets (12 mm diameter, 2.0-2.5 mm thickness) with a uniaxial pressure of $150 \mathrm{MPa}$ and sintered at $1450{ }^{\circ} \mathrm{C}$ for 10 hours. The experimental density of the samples was measured by the Archimedes method in distilled water. The crystallographic phase composition was verified by X-ray powder diffraction (XRD) technique (Bruker D8, Germany). The lattice parameter $\left(\mathrm{a}_{0}\right)$ was estimated using the least-square extrapolation method, as described by Pramanick et al. [23]. The microstructure was characterized by a high-resolution scanning electron microscopy (SEM, Zeiss Merlin, Germany). The grain size of the samples was 
measured using a linear intercept method multiplying with correction factor 1.57 [24]. The electrical conductivity of the samples was examined in the range of $250-500{ }^{\circ} \mathrm{C}$ in $25^{\circ} \mathrm{C}$ intervals by impedance spectroscopy (Solarton 1260, UK) in a frequency range of $0.01 \mathrm{~Hz}$ to $10 \mathrm{MHz}$ with an applied $100 \mathrm{mV}$ alternating current signal under ambient air. The sample has a rectangular dimension and is symmetrically coated with silver paste (No-VOCs, SPI, USA), dried at $500{ }^{\circ} \mathrm{C}$ for 1 hour. The resultant impedance data is fitted by employing an equivalent circuit model and analyzed by ZView software. For the electromechanical response, the strain was evaluated with a proximity sensor of the capacitive type (Lion Precision, USA) with lock-in detection, as explained in detail elsewhere [8]. The bar shape sample (thickness $\sim 0.8-1.0 \mathrm{~mm}$ ) was sandwiched between two metal electrodes made of aluminium (top) and brass (bottom). The top electrode was mechanically attached to the sample during the measurement applying force of $1 \mathrm{~N}$.

\section{Results and Discussion}
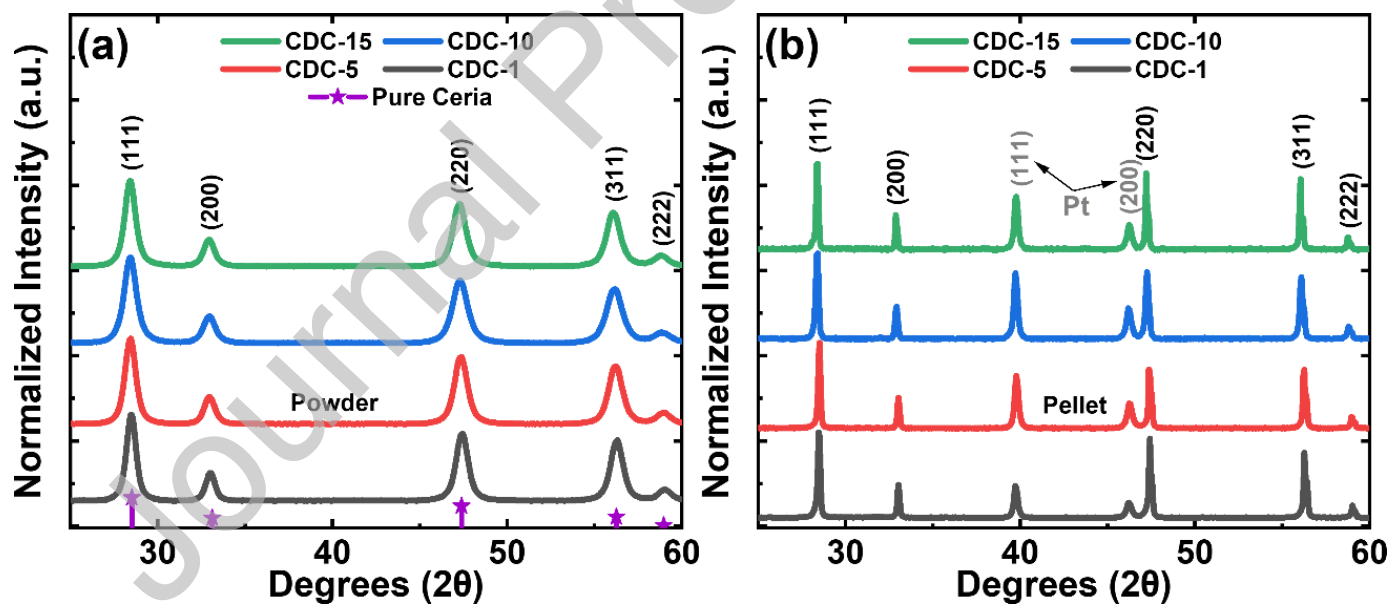

Figure 1: X-ray diffraction (XRD) pattern of the CDC $\left(\mathrm{Ce}_{1-x} S m_{x / 2} N d_{x / 2} \mathrm{O}_{2-x / 2}\right)$ (a) powders and (b) sintered pellets, where $x=0.01-0.15$. The results are compared with pure ceria ICSD \# 251473.

The X-ray diffraction (XRD) patterns of CDC powders and respective sintered pellets are shown in Fig. 1. All samples exhibit a characteristic single-phase cubic fluorite crystal structure $(F m-3 m)$. Considering the detection limits of the XRD method (5-7 \% in volume), no other impurity diffraction peaks are evidenced. Moreover, the increment of dopant 
concentration shifted the featured peaks, e.g. (111) toward lower $2 \theta$ angles, confirming the expansion of the lattice parameter to dopant concentration.

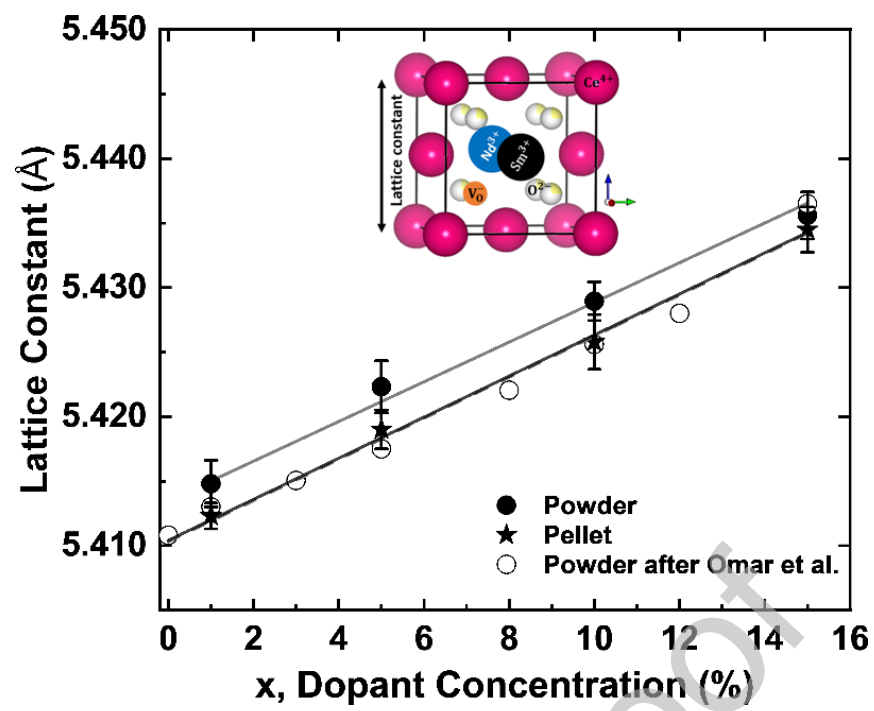

Figure 2: Estimated lattice constant as a function of total dopant concentration (mol\%) where $\mathrm{x}=$ 0.01-0.15. Results are compared with the powder data of Omar et al. [16]. Inset: Schematic representation of a typical unit cell of ceria lattice in the presence of trivalent co-dopants.

The assessed lattice constant of both the starting powders and sintered pellets as a function of total dopant content is illustrated in Fig. 2, which shows that the lattice constant increases linearly with the concentration of dopant, according to Vegard's rule [25]. A similar linear trend was previously demonstrated by Omar et al. in Sm/Nd-co-doped ceria system [16][17]. Moreover, other reports describe that the lattice constant of the singly doped-ceria system follows a quadratic expression with dopant concentration where the coefficient of the second-order is rendered as oxygen vacancy ordering [26][27][28]. The absence of secondorder term underlines that dopant associated short-range oxygen vacancy ordering in the CDC sample is relatively less in comparison with singly-doped ceria, as demonstrated in other earlier work [16]. Besides, lattice constant decreases considerably for the sintered pellet. Such an outcome is due to the fact that long-term thermal treatment at higher temperatures, i.e., $1450{ }^{\circ} \mathrm{C}$ for 10 hours, initiates the lattice defect annihilation during the sintering and densification process by the mass transport mechanism [29][30][31]. 

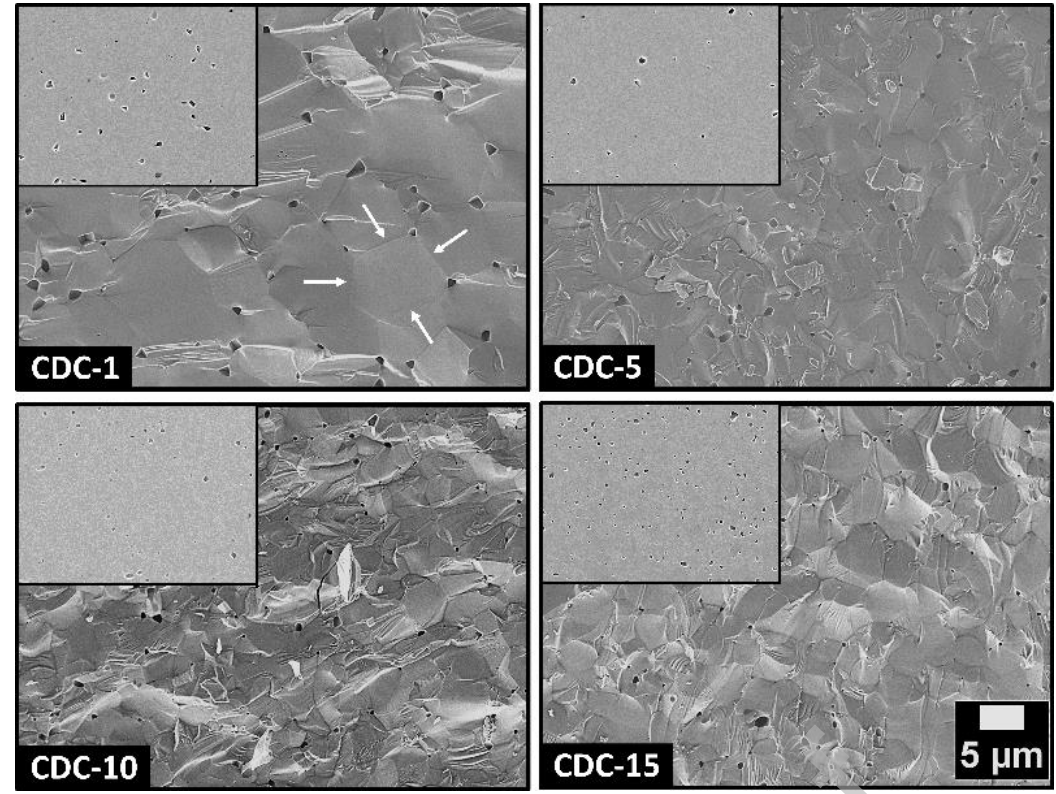

Figure 3: The SEM micrographs of the cold-fractured and polished cross-sectional surface of the sintered pellets. All samples have the same scale bar of $5 \mu \mathrm{m}$.

The relative density of all sintered pellets was found to be above $95 \%$, in agreement with the observed microstructural results in Fig. 3, where only a few percents of isolated residual porosity was observed. The average grain size of all samples ranges in-between 3-6 $\mu \mathrm{m}$, showing a reducing trend with increasing dopant content. Most of these grains have equilibrium shape, small residual grain boundary curvature (white arrows), and are thermodynamically relaxed.

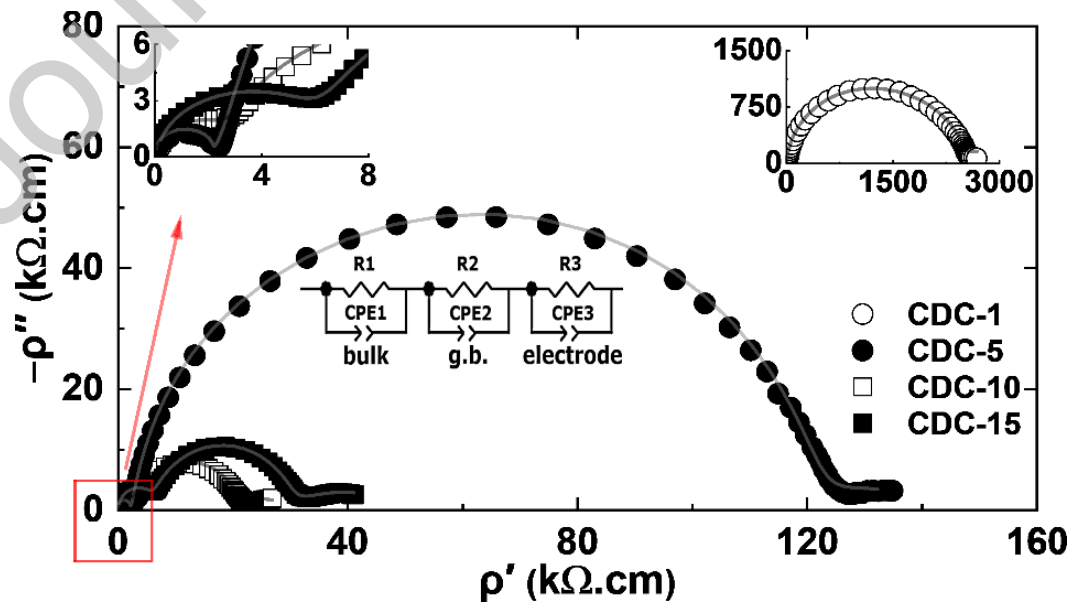

Figure 4: Illustration of geometry normalized Nyquist plots ( $\rho^{\prime}$ vs $\left.\rho "\right)$ for CDC pellets, examined in the air at $300^{\circ} \mathrm{C}$. 
The characterization of the ionic electrical properties at low temperatures identifies transport mechanisms of oxygen defect in the materials as a result of association and blocking effects at the bulk/grain boundary. Particularly, electrochemical impedance spectroscopy at moderately high frequency allows separating such contributions. The results are presented in Fig. 4, as analyzed by the method described in Ref. [18][19]. It is worth remarking that, despite being generally associated with the grain boundaries, the ionic blocking effect can also arise from other chemical and microstructural factors, e.g., porosity, nanodomain formation, impurity/dopant segregation, etc. [32][33][34]. In Fig. 4, the geometry normalized Nyquist plots at $300{ }^{\circ} \mathrm{C}$ exhibit two well-defined semicircles that correspond to high and intermediate frequency attributed bulk and grain boundary resistance of the co-doped samples, respectively. The low-frequency arc refers to the electrolyte-electrode polarization, and it is not relevant in this work. These Nyquist plots were fitted by parallel equivalent circuits of $R Q$ elements where $R$ and $Q$ are denoted respectively as resistor and constant phase element. As observed, the bulk resistance differs moderately among the samples, however, a prominent deviation is measured in grain boundary contribution, especially for CDC-1 and CDC-5 compound. Both of them display a grain boundary blocking factor $\left(\alpha_{\text {g.b. }}\right)$ about $\approx 0.98$ at $300^{\circ} \mathrm{C}$, which is the ratio of grain boundary resistance to the total resistance $\left(\alpha_{\text {g.b. }}=\frac{\mathrm{R}_{\text {g.b. }}}{\mathrm{R}_{\text {bulk }}+\mathrm{R}_{\text {g.b. }}}\right)$ [35]. The high ion-blocking effect also decreases the characteristic frequency response of the grain boundary (see Table 1).

The Arrhenius plot in Fig. 5 illustrates the bulk (grain) and (specific) grain boundary electrical conductivity $\left(\sigma=1 / \rho^{\prime}\right)$ for all samples over the temperature ranges of $250-400{ }^{\circ} \mathrm{C}$. For the estimation, conductivity accounts the actual volume of bulk and grain boundary, as reported in Ref. [36]. Noteworthy is the pronounced difference between these two conduction mechanisms, with the value of bulk conductivity typically exceeds a factor of two-four larger than grain boundary. In Fig. 5a, when comparing bulk conductivity between the samples, conductivity increases with dopant concentration for low doping ( $<5 \mathrm{~mol} \%)$ and slightly falls off for the highly doped system. Such a result is attributed to the fact that the highly doped 
compositions, e.g., CDC-10 and higher, possibly develop a cation-defect association interaction, which not only minimizes free oxygen vacancy concentration but also decreases the number of low energy migration paths for diffusion by forming high migration energy barriers [15][37]. This finding is in agreement with the enhanced activation energy value for bulk (see Table 1). A preceding experimental study by Omar and co-workers for the grain ionic conductivity of $\mathrm{Ce}_{1-\mathrm{x}} \mathrm{Sm}_{\mathrm{x} / 2} \mathrm{Nd}_{\mathrm{x} / 2} \mathrm{O}_{2-\mathrm{x} / 2}$ exhibits a parallel trend [16]. The hypothesis of dopant-defect association in oxygen defective cerium oxides is well-consolidated for $\mathrm{Gd}, \mathrm{Nd}$, Sm- and other substitutional doping [38].

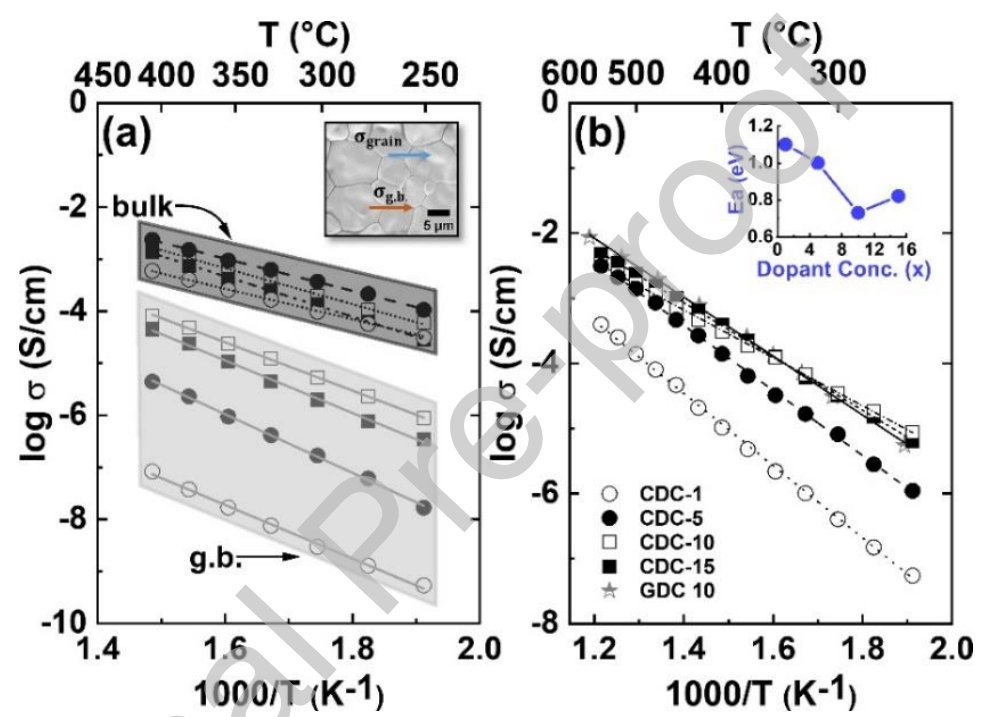

Figure 5: Arrhenius plot for the estimation of (a) bulk and (specific) grain boundary, (b) total, electrical conductivities of sintered CDC pellets. The GDC-10 sample (sintered at $1450{ }^{\circ} \mathrm{C}$ for 10 hours) is taken from Ref. [9].

The ion blocking effect was assumed to be dominated by the grain boundary, which is strongly influenced by nominal dopant concentration. The grain boundary conductivity indicates an incremental trend until 10 mol\% doping then marginally decreases for the CDC15 sample. According to most literature reports, grain boundary conductivity increases with dopant concentration because of the decreasing trend of space charge potential as a function of nominal dopant content [39][36]. The in-depth mechanism of blocking effect is challenging to explain exclusively via electrochemical measurements since it can include other features such as dopant segregation effects at the grain boundary or nanodomain 
formation, as mentioned above [30][31][33]. From the Arrhenius plot in Fig. 5a, the activation energy for grain boundary conductivity is estimated at around $1.0 \mathrm{eV}$, which is typical for the ceria compound.

The total conductivity is affected by the co-operative effects of both bulk and grain boundary. The plot in Fig. 5b displays that total electrical conductivity increases with dopant concentration. The inset of this plot represents the activation energy for total conductivity, exhibiting a higher value for the sample with low dopant concentration. Such an outcome is possibly associated with the significant ion blocking barrier effects governed in these compounds. The total conductivity of CDC-10, CDC-15, and reference GDC-10 sample have comparable values, an order of magnitude higher than CDC-5, particularly in the lowtemperature regime $\left(<375^{\circ} \mathrm{C}\right)$. In summary, the impedance analysis shows that at a fixed thermal treatment, i.e., $1450{ }^{\circ} \mathrm{C}$ for 10 hours in this experiment results in a large ion blocking effect for dilute doping. The blocking factor scales down with increasing nominal dopant content. A direct comparison with GDC-10 (sintered at the same condition), the CDC-10 sample represents a reduced blocking effect.

Table 1: The average grain size and summary of the impedance analysis for the CDC ceramics, in comparison with the GDC-10 sample sintered at the same condition [9].

\begin{tabular}{|c|c|c|c|c|c|c|}
\hline $\begin{array}{c}\text { Sample } \\
\text { ID }\end{array}$ & $\begin{array}{c}\mathbf{E}_{\text {grain }} \\
(\mathbf{e V})\end{array}$ & $\begin{array}{c}\mathbf{E}_{\text {g.b. }} \\
(\mathbf{e V})\end{array}$ & $\begin{array}{c}\sim \mathbf{f}_{\text {grain }} \\
\mathbf{( H z})\end{array}$ & $\begin{array}{c}\sim \mathbf{f}_{\text {g.b. }} \\
\mathbf{( H z )}\end{array}$ & $\begin{array}{c}\boldsymbol{\alpha}_{\text {g.b. }} \text { at } \mathbf{3 0 0} \\
{ }^{\circ} \mathbf{C}\end{array}$ & $\begin{array}{c}\sim \text { Grain Size } \\
(\boldsymbol{\mu m})\end{array}$ \\
\hline CDC-1 & 0.58 & 1.0 & $1.0 \cdot 10^{6}$ & $1.5 \cdot 10^{1}$ & 0.99 & $5.6 \pm 0.3$ \\
\hline CDC-5 & 0.60 & 1.10 & $2.0 \cdot 10^{6}$ & $3.0 \cdot 10^{2}$ & 0.98 & $3.7 \pm 0.5$ \\
\hline CDC-10 & 0.68 & 0.90 & $1.0 \cdot 10^{5}$ & $1.0 \cdot 10^{3}$ & 0.80 & $3.4 \pm 0.4$ \\
\hline CDC-15 & 0.76 & 1.0 & $8.0 \cdot 10^{4}$ & $1.0 \cdot 10^{3}$ & 0.75 & $3.0 \pm 0.2$ \\
\hline GDC-10 & 0.65 & 0.95 & $2.0 \cdot 10^{5}$ & $1.5 \cdot 10^{3}$ & 0.90 & $2.0 \pm 0.3$ \\
\hline
\end{tabular}




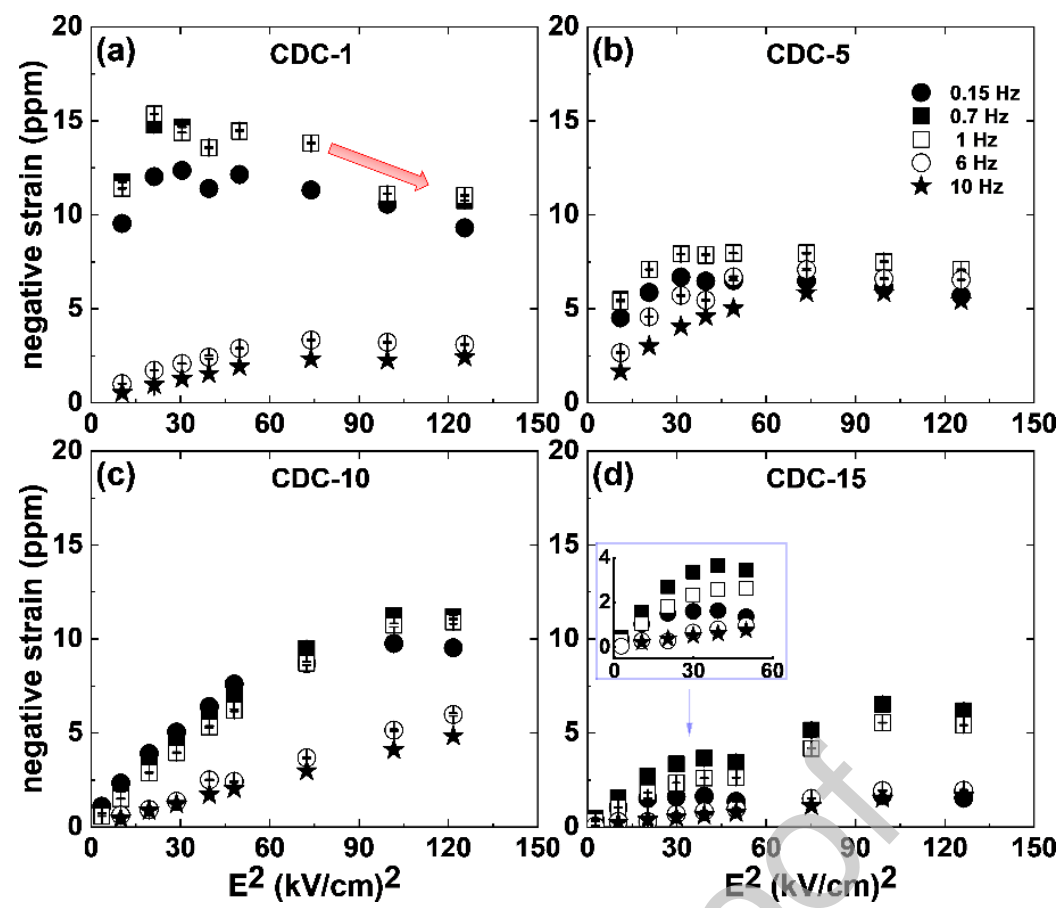

Figure 6: (a)-(d) The electrostrictive negative strain as a function of applied electric field square at frequencies between $0.15-10 \mathrm{~Hz}$. The error bar is smaller than the symbol size.

The electrostrictive strain with a response to the square of the applied electric field at selected frequencies (up to $10 \mathrm{~Hz}$ ) is demonstrated in Fig. 6. As expected, all CDC samples exhibit a negative longitudinal strain at the second harmonic of the electric field, in agreement with the previously published reports of GDC bulk materials [8][9][14]. The observed electromechanical strain value starts saturating at a particular electric field and unexpectedly, in a few cases, decreases at higher electric fields (see pink arrow in Fig. 6). Additionally, the figures also display that with increasing frequency, the magnitude of electromechanical strain declines significantly. Interestingly, both the CDC-10 and CDC-15 samples follow the linear trend of electrostriction strain vs $E^{2}$ for 6 and $10 \mathrm{~Hz}$ in the entire range of investigated electric field. The lack of strain saturation at these frequencies could be associated with the low blocking barrier effect, formed in these compositions. Despite inconclusive, such findings are consistent with previous report [9]. For example, the microcrystalline bulk GDC revealed no strain saturation when the blocking factor is noticeably low, e.g., $\approx 0.65$. 

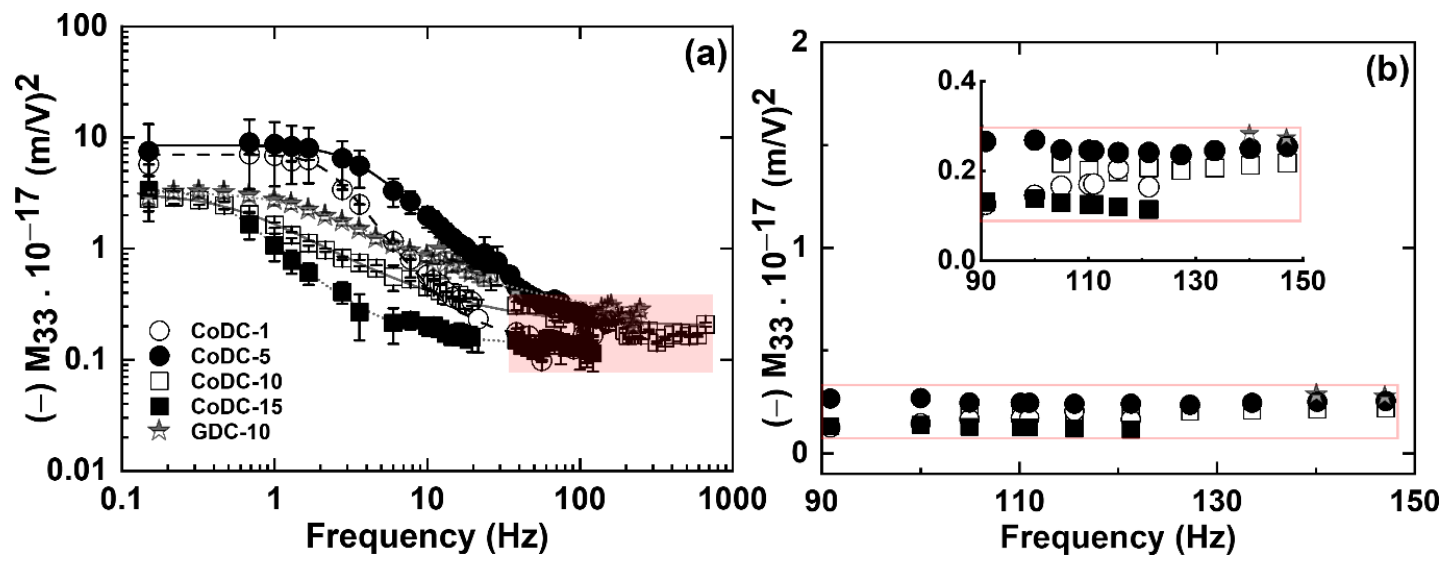

Figure 7: (a) The electrostrictive strain coefficient $\left(\mathrm{M}_{33}\right)$ as a function of applied frequencies ranging from $0.15-700 \mathrm{~Hz}$. The results are compared with the GDC-10 sample from Ref. [9]. (b) The value of $\mathrm{M}_{33}$ at a high-frequency regime.

The electrostriction strain coefficient $\left(\mathrm{M}_{33}\right)$ is strongly related to the applied field frequency and represented in Fig. 7.a. Remarkably, all CDC samples display non-ideal Debye type relaxation and are fitted with the following relation [8]:

$$
M_{33}(f)=\frac{M_{33}^{0}}{\sqrt{1+(\tau . f)^{2+\alpha}}}+M_{33}^{\infty}
$$

(4)

Where $\mathrm{M}_{33}^{0}$ and $\mathrm{M}_{33}^{\infty}$ are the electrostriction coefficient at low and high frequencies, respectively. $\tau$ and $\alpha$ are denoted as relaxation time and non-ideality factor, correspondingly. The fitting parameters from Eqn (4) are presented in Table 2. In the low-frequency regime, for instance below $10 \mathrm{~Hz}$, both the CDC-1 and CDC-5 samples exhibit considerably higher $\mathrm{M}_{33}$ values than of highly doped $\mathrm{CDC}-10$ and $\mathrm{CDC}-15$. These results emphasize that the electrostriction mechanism in defective ceria does not certainly necessitate a large concentration of oxygen vacancies. The former compounds have not only low oxygen vacancy concentration but also a high ion blocking factor compared to the latter. The lowfrequency $M_{33}$ values and blocking barrier factor of the GDC-10 sample lies in between such results. From this perspective, the current findings emphasize that $\mathrm{M}_{33}$ value in the lowfrequency regime is strongly dominated by the ion-blocking barrier factor developed in the materials. The ion blocking barriers are usually controlled by microstructural features (grain size) and especially by the effective dopant distribution, as shown in the previous study [9]. 
Moreover, at higher frequencies, such as above $50 \mathrm{~Hz}$, the magnitude of $\mathrm{M}_{33}$ is relatively constant (shaded box in Fig. 7.a) and very close to each sample. The high-frequency electrostriction value is illustrated in a separate plot in Fig. 7.b. The $\mathrm{M}_{33}$ varies in between 0.1-0.3 $\cdot 10^{-17}(\mathrm{~m} / \mathrm{V})^{2}$ for all samples and is independent of both oxygen vacancy concentration and configuration. Above all, the estimated $\mathrm{M}_{33}$ value for all examined samples at the high-frequency regime lies in the order of $\approx 10^{-18}(\mathrm{~m} / \mathrm{V})^{2}$, a value that is still one order of magnitude larger than what Newnham's classical model predicts.

Table 2: Summary of the fitting parameters of Eqn 4, in comparison with GDC-10 ceramics [9].

\begin{tabular}{|c|c|c|c|c|}
\hline Sample ID & $\begin{array}{c}(-) \mathbf{M}_{33}^{\mathbf{0}}, \mathbf{1 0}^{-\mathbf{1 7}} \\
(\mathbf{m} / \mathbf{V})^{2}\end{array}$ & $\begin{array}{c}(-) \mathbf{M}_{33}^{\infty}, \mathbf{1 0}^{-17} \\
(\mathbf{m} / \mathbf{V})^{2}\end{array}$ & $\boldsymbol{\tau}, \mathbf{S}$ & $\boldsymbol{\alpha}$ \\
\hline CDC-1 & $6.9 \pm 0.23$ & $0.15 \pm 0.02$ & $0.45 \pm 0.03$ & $2.0 \pm 0.3$ \\
\hline CDC-5 & $8.3 \pm 1.3$ & $0.20 \pm 0.06$ & $0.30 \pm 0.01$ & $0.70 \pm 0.1$ \\
\hline CDC-10 & $2.9 \pm 0.05$ & $0.19 \pm 0.01$ & $1.8 \pm 0.1$ & $-0.3 \pm 0.1$ \\
\hline CDC-15 & $3.3 \pm 0.2$ & $0.13 \pm 0.01$ & $2.4 \pm 0.05$ & $0.8 \pm 0.06$ \\
\hline GDC-10 & $3.0 \pm 0.1$ & $0.20 \pm 0.04$ & $0.7 \pm 0.05$ & $-0.3 \pm 0.1$ \\
\hline
\end{tabular}

\section{Conclusion}

In this work, oxygen defective ceria compounds were synthesized by a co-doping strategy with a composition of $\mathrm{Ce}_{1-\mathrm{x}} \mathrm{Sm}_{\mathrm{x} / 2} \mathrm{Nd}_{\mathrm{x} / 2} \mathrm{O}_{2-\mathrm{x} / 2}$ where $\mathrm{x}=0.01-0.15$ and sintered at higher temperatures. The equimolar co-doping minimizes the short-range oxygen vacancy ordering in the lattice. Moreover, all samples develop a different ion blocking effect, which in turn depends on the nominal oxygen vacancy concentration and their configuration. These materials exhibit non-classical electrostriction with an unusual strain saturation and relaxation mechanisms as a function of applied electric field amplitude and frequency. For instance, a large electrostrictive coefficient $\geq 10^{-17}(\mathrm{~m} / \mathrm{V})^{2}$ is reported at a low frequency under an applied electric field below $5 \mathrm{kV} / \mathrm{cm}$. In particular, the electrostriction value at the low-frequency regime maintains a strict relationship with oxygen vacancy configuration at the blocking barrier. Electrostriction at high-frequency regime expresses neither concentration nor configuration dependency but a steady value. In view of this, we conclude that low ion 
blocking barrier effects might be favorable to obtain a constant $\mathrm{M}_{33}$ value for a wide range of frequencies.

\section{Acknowledgments}

This research was supported by DFF-Research project grants from the Danish Council for Independent Research, Technology and Production Sciences, June 2016, grant number 48293 (GIANT-E), and the European H2020-FETOPEN-2016-2017 project BioWings, grant number 801267.

\section{References}

[1] R. E. Newnham, V. Sundar, R. Yimnirun, J. Su, and Q. M. Zhang, "Electrostriction: nonlinear electromechanical coupling in solid dielectrics," J. Phys. Chem. B, 101 (1997) 10141-10150.

[2] A. L. Kholkin, E. K. Akdogan, A. Safari, P. F. Chauvy, and N. Setter, "Characterization of the effective electrostriction coefficients in ferroelectric thin films," J. Appl. Phys., 89 (2001) 8066.

[3] D. J. Laser and J. G. Santiago, "A review of micropumps," Journal of Micromechanics and Microengineering, 14 (2004), R35-R64.

[4] R. Yimnirun, S. M.-L. Eury, V. Sundar, P. J. Moses, S.-J. Jang, and R. E. Newnham, "Electrostriction measurements on low permittivity dielectric materials," J. Eur. Ceram. Soc.,19 (1999) 1269-1273.

[5] S. Eury, R. Yimnirun, V. Sundar, P. J. Moses, S. J. Jang, and R. E. Newnham, "Converse electrostriction in polymers and composites," Mater. Chem. Phys., 61 (1999) 18-23.

[6] N. Yavo et al., "Large Nonclassical Electrostriction in $(\mathrm{Y}, \mathrm{Nb})$-Stabilized $\delta-\mathrm{Bi}_{2} \mathrm{O}_{3}$," Adv. Funct. Mater., 26 (2016) 1138-1142.

[7] R. Korobko, A. Patlolla, A. Kossoy, E. Wachtel, H. L. Tuller, A. I. Frenkel, and I. Lubomirsky, "Giant electrostriction in Gd-doped ceria," Adv. Mater., 24 (2012) 5857-5861.

[8] N. Yavo, O. Yeheskel, E. Wachtel, D. Ehre, A. I. Frenkel, and I. Lubomirsky, "Relaxation and saturation of electrostriction in 10 mol\% Gd-doped ceria ceramics," Acta Mater., 144 (2018) $411-418$

[9] A. Kabir et al., "Effect of oxygen defects blocking barriers on gadolinium doped ceria (GDC) electro-chemo-mechanical properties," Acta Mater., 174 (2019) 53-60.

[10] Y. Li, O. Kraynis, J. Kas, T. Weng, D. Sokaras, R. Zacharowich, I. Lubomirsky, and A. I. Frenkel, "Geometry of electromechanically active structures in Gadolinium - Doped Cerium oxides," AIP Adv., 6 (2016) 055320-7.

[11] O. Kraynis, J. Timoshenko, J. Huang, H. Singh, E. Wachtel, A. I. Frenkel and I. Lubomirsky, "Modeling Strain Distribution at the Atomic Level in Doped Ceria Films with Extended X-ray Absorption Fine Structure Spectroscopy," Inorg. Chem., 58 (2019) 7527-7536.

[12] R. Korobko, A. Lerner, Y. Li, E. Wachtel, A. I. Frenkel, and I. Lubomirsky, "In-situ extended Xray absorption fine structure study of electrostriction in Gd doped ceria," Appl. Phys. Lett., 106 (2015) 042904.

[13] M. Hadad, H. Ashraf, G. Mohanty, C. Sandu, and P. Muralt, "Key-features in processing and microstructure for achieving giant electrostriction in gadolinium doped ceria thin films," Acta Mater., 2016 (118) 1-7. 
[14] A. Kabir, J. Kyu Han, B. Merle, and V. Esposito, "The role of oxygen defects on the electrochemo-mechanical properties of highly defective gadolinium doped ceria," Mater. Lett., 266 (2020) 127490.

[15] I. A. Abrikosov, S. I. Simak, D. A. Andersson, N. V. Skorodumova, and B. Johansson, "Optimization of ionic conductivity in doped ceria," Proc. Natl. Acad. Sci., 103 (2006) 35183521, 2006.

[16] S. Omar, E. D. Wachsman, and J. C. Nino, "Higher conductivity $\mathrm{Sm}^{3+}$ and $\mathrm{Nd}^{3+}$ co-doped ceria-based electrolyte materials," Solid State lonics, 178 (2008) 1890-1897.

[17] S. Omar, E. D. Wachsman, and J. C. Nino, "Higher ionic conductive ceria-based electrolytes for solid oxide fuel cells," Appl. Phys. Lett., 91 (2007) 144106.

[18] M. G. Bellino, D. G. Lamas, and N. E. W. De Reca, "Enhanced ionic conductivity in nanostructured, heavily doped ceria ceramics," Adv. Funct. Mater., 16 (2006) 107-113.

[19] S. M. Haile, D. L. West, and J. Campbell, "The role of microstructure and processing on the proton conducting properties of gadolinium-doped barium cerate," J. Mater. Res., 13 (1998) 1576-1595.

[20] V. Esposito and E. Traversa, "Design of electroceramics for solid oxides fuel cell applications: Playing with ceria," J. Am. Ceram. Soc., 91 (2008) 1037-1051.

[21] J. Fleig and J. Maier, "The impedance of ceramics with highly resistive grain boundaries: Validity and limits of the brick layer model," J. Eur. Ceram. Soc., 19 (1999) 693-696.

[22] J. Fleig, "The grain boundary impedance of random microstructures: Numerical simulations and implications for the analysis of experimental data," in Solid State lonics, 150 (2002) 181193.

[23] A. Pramanick, S. Omar, J. C. Nino, and J. L. Jones, "Lattice parameter determination using a curved position-sensitive detector in reflection geometry and application to $\mathrm{Sm}_{\mathrm{x} / 2} \mathrm{Nd}_{\mathrm{x} / 2} \mathrm{Ce}_{1-\mathrm{x}} \mathrm{O}_{2-\delta}$ ceramics," J. Appl. Crystallogr., 42 (2009) 490-495.

[24] H. Abrams, "Grain size measurement by the intercept method," Metallography, 4 (1971) 59-78.

[25] L. Vegard, "Die Konstitution der Mischkristalle und die Raumfüllung der Atome," Zeitschrift für Phys., 5 (1921) 17-26.

[26] I. STEPHENS and J. KILNER, "Ionic conductivity of $\mathrm{Ce}_{1-\mathrm{x}} \mathrm{Nd}_{x} \mathrm{O}_{2-x / 2}$," Solid State lonics, 177 (2006) 669-676.

[27] R. D. Shannon, "Revised effective ionic radii and systematic studies of interatomic distances in halides and chalcogenides," Acta Crystallogr. Sect. A, A32 (1976) 751-767.

[28] J.M. Bevan, W.W. Barker, T.C. Parks, in: L. Eyring (Ed), Proceedings of the fourth conference on rare earth research, Gordon and Breach, New York, (1965) 441.

[29] V. Esposito et al., "Accelerated ceria-zirconia solubilization by cationic diffusion inversion at low oxygen activity," J. Mater. Chem. A, 4 (2016) 16871-16878.

[30] F. Teocoli, D. W. Ni, S. Sanna, K. Thydén, F. C. Fonseca, and V. Esposito, "Fast mass interdiffusion in ceria/alumina composite," J. Mater. Chem. A, 3 (2015) 17135-17143.

[31] D. W. Ni, D. Z. de Florio, D. Marani, A. Kaiser, V. B. Tinti, and V. Esposito, "Effect of chemical redox on Gd-doped ceria mass diffusion," J. Mater. Chem. A, 3 (2015) 18835-18838.

[32] V. Esposito, M. Zunic, and E. Traversa, "Improved total conductivity of nanometric samariadoped ceria powders sintered with molten $\mathrm{LiNO}_{3}$ additive," Solid State lonics, 180 (2009) 1069-1075.

[33] Z. P. Li, T. Mori, G. J. Auchterlonie, J. Zou, and J. Drennan, "Direct evidence of dopant segregation in Gd-doped ceria," Appl. Phys. Lett., 98 (2011) 093104. 
[34] X. Guo and R. Waser, "Electrical properties of the grain boundaries of oxygen ion conductors: Acceptor-doped zirconia and ceria," Prog. Mater. Sci., 51 (2006) 151-210.

[35] K. Neuhaus, R. Dolle, and H.-D. Wiemhöfer, "Assessment of the effect of transition metal oxide addition on the conductivity of commercial Gd-Doped Ceria," J. Electrochem. Soc., 165 (2018) F533-F542.

[36] H. J. Avila-Paredes, K. Choi, C.-T. Chen, and S. Kim, "Dopant-concentration dependence of grain-boundary conductivity in ceria: A space-charge analysis," J. Mater. Chem., 19 (2009) 4837.

[37] R. Krishnamurthy, Y.-G. Yoon, D. J. Srolovitz, and R. Car, "Oxygen Diffusion in YttriaStabilized Zirconia: A New Simulation Model,” J. Am. Ceram. Soc., 87 (2005) 1821-1830.

[38] H. Inaba and H. Tagawa, "Ceria-based solid electrolytes," Solid State lonics, 83 (1996) 1-16.

[39] T. S. Zhang, J. Ma, L. B. Kong, S. H. Chan, and J. A. Kilner, "Aging behavior and ionic conductivity of ceria-based ceramics: A comparative study," Solid State Ionics, 170 (2004) 209-217. 
Graphical abstract

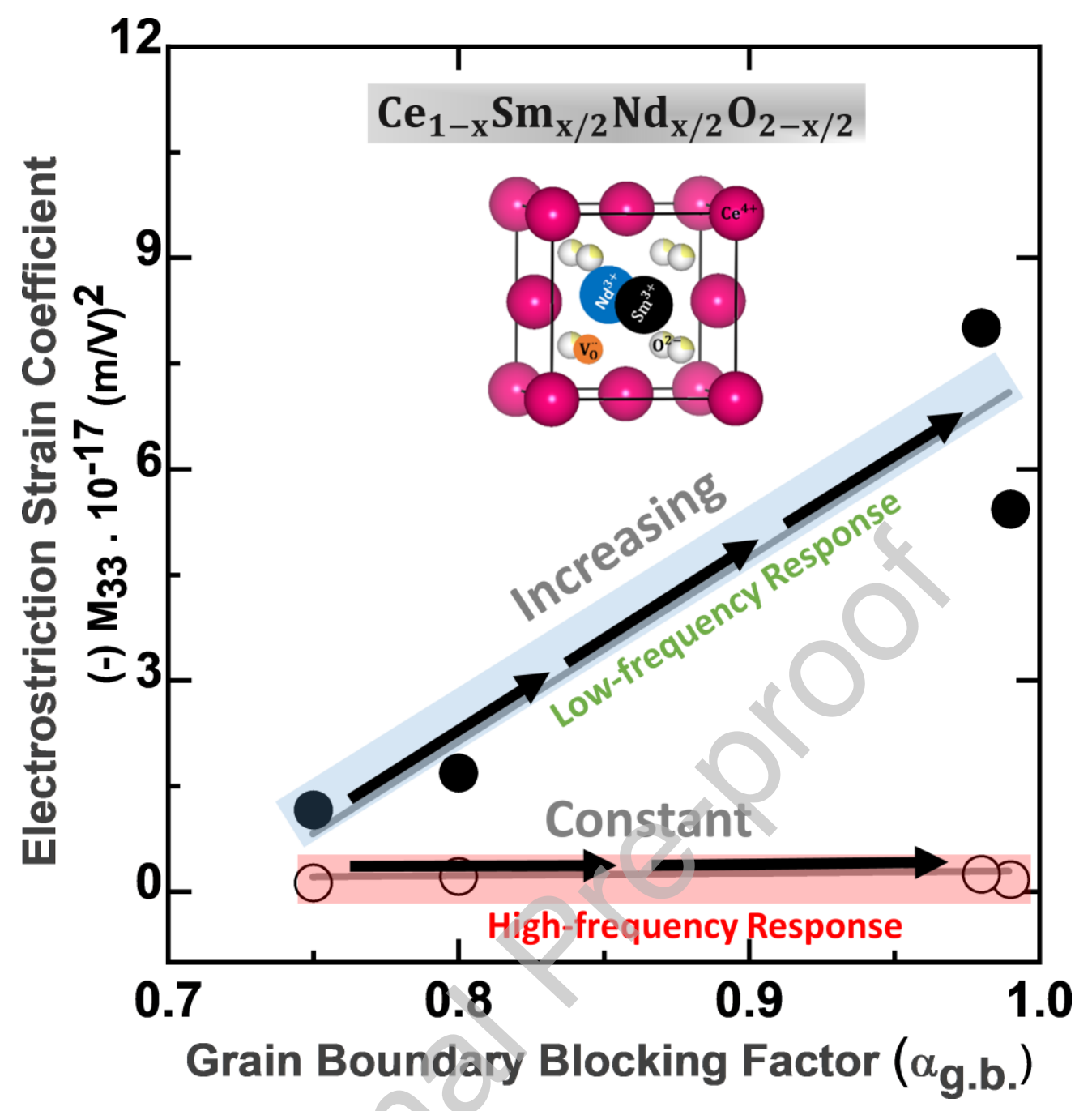

